can be identified as the contribution of the $n$th aperture to the field at $(\rho, \phi)$. We can say that the array of $N$ horn apertures is focused to the point $(\rho, \phi)$ when the phase of each contribution $E_{z, n}$ is identical. An equivalent statement is that

$$
\delta_{n}=- \text { phase of } \sum_{m=-\infty}^{+\infty} f_{m} V_{m} I_{m}\left(\gamma_{1} \rho\right) e^{-t m\left(\phi-\phi_{n}\right)}
$$

The choice of the amplitude factor $\Delta_{n}$ is still open. The coefficient $f_{m}$ is defined by (18) and involves the electrical properties of the target and the concentric buffer region. In the case where the latter is absent or vanishing small (i.e., $b \rightarrow a$ ), we see that

$$
f_{m}=\left[I_{m}\left(\gamma_{1} a\right)\right]^{-1} \text {. }
$$

The other key coefficient in (30) is $V_{m}$, which depends on the common field distribution in a given aperture. Assuming that the horns are excited only by a TEM (transverse electromagnetic) mode, it is reasonable to assume a cosinusoidal field distribution at the aperture which vanishes at the side walls. Thus, we may adopt the form

$$
\hat{E}_{z}(\hat{\phi})=E_{0} \cdot \cos \left(\frac{N \hat{\phi}}{2}\right)
$$

for the range $-\frac{\pi}{N}<\hat{\phi}<\frac{\pi}{N}$. Then, using (27), we see that

$$
\begin{aligned}
V_{m} & =b E_{0} \int_{-\pi N}^{\pi / N} \cos \left(\frac{N \hat{\phi}}{2}\right) e^{i m \hat{\phi}} d \hat{\phi} \\
& =b E_{0} \cos \left(m \frac{\pi}{N}\right) \frac{N}{(N / 2)^{2}-m^{2}} \\
& =b E_{0} \pi / N \quad \text { for } m=N / 2 .
\end{aligned}
$$

All quantities on the right-hand side of (30) are now specified, and the phase factors $\delta_{n}$ may be calculated for any desired point $(\rho, \phi)$. We are still free to select the amplitude factors $\Delta_{n}$ if additional constraints are made.

\section{Final Remarks}

There are a number of generalizations and extensions of the present model that we might mention. The case where the target (defined by $\rho<a$ in Fig. 1) is concentrically layered presents no difficulty in analysis. Here, we can use the present formulation provided $Y_{1, m}$ (the surface admittance) of mode of order $m$ at $\rho=a$ is replaced by the appropriate form for the equivalent nonuniform transmission line [8]. Another extension is to allow explicity for the limited axial extent (in the $z$-direction) of the apertures and to include the axial variation of the fields [8],[10]. Now we must allow for the intrinsic hybrid nature of the fields because the TE (transverse electric) and TM (transverse magnetic) modes are coupled [8]. At the same time, both the axial and the azimuthal (i.e., $z$ and $\phi$, respectively) variations of the aperture fields can be accounted for. In a further extension, we can consider the influence of the finite length of the cylindrical target by imposing a zero axial current flow condition at the bounding planes (top and bottom) [11].

There is another interesting concept that we might also mention in the present context. In order to "focus" the array, we adopted a procedure whereby the fields of the individual apertures were selected to have a phase such that all contributions at the internal field point were additive. Now we could have turned the problem around and started with a hypothetic electric line source at the internal point and deduce the corresponding re- ceived signal in each aperture. This would lead to an alternative but equivalent procedure to deduce the phase angles $\delta_{n}$ needed to design the focused aperture. This latter approach could be adapted to more complicated target geometries where purely numerical methods would be required to solve the electromagnetic problem. However, in the interim, it would seem prudent to restrict attention to analytically viable models if insight and understanding are desired. We are currently undertaking such a study and the results will be reported in Part II.

\section{ACKNOWLEDGMENT}

The author wishes to thank Dr. T. C. Cetas for his encouragement and support of this study.

\section{REFERENCES}

[1] N. Morita and J. B. Andersen, "Near-field absorption in a circular cylinder from electric and magnetic line sources," Bioelectromagnetic, vol. 3, pp. 253-274, 1982

[2] J. R. Oleson, T. C. Cetas, and P. M. Cory, "Hyperthermia by magnetic induction: Experimental and theoretical results for coaxial coil pairs," Radiation Res., vol 96, pp. 175-186, 1983.

[3] P. F. Turner, "Regional hyperthermia with an annular phased array," IEEE Trans. Bio-Med. Eng., vol. BME-31, pp. 106-114, 1984.

[4] G. Arcangeli, P. P. Lombardini, G. A. Lovisolo, G. Marsiglia, and M Piatteli, "Focussing of $915 \mathrm{MHz}$ electromagnetic power on deep human tissues," IEEE Trans. Bto-Med. Eng., vol. BME-31, pp. 47-52, 1984.

[5] W. Gee, S. W-. Lee, N. K. Bong, C. A. Cain, R. Mittra, and R. L. Magin, "Focussed array hyperthermia applicator." IEEE Trans. Bio-Med. Eng., vol. BME-31, pp. 38-46, 1984.

[6] J. R. Oleson, "A review of magnetic induction methods for hyperthermia treatment of cancer," IEEE Trans. Bio-Med. Eng., vol. BME-31, pp. 91-97, 1984.

[7] J. R. Wait, Electromagnetic Radiation from Cylindrical Structures. London: Pergamon, 1959.

[8] J. R. Wait, Electromagnetic Wave Theory. New York: Harper and Row, 1984.

[9] M. Abramowitz and I. Stegun, Handbook of Mathematical Functions. New York: Dover, 1975.

[10] H. S. Ho, A. W. Guy, R. A. Sigelmann, and J. F. Lehmann, "Microwave heating of simulated limbs by aperture sources," IEEE Trans. Microwave Theory Tech., vol. MTT-19, pp. 224-231, 1971.

[11] J. R. Wait and D. A. Hill, "Excitation of a homogeneous conductive cylinder of finite length by a prescribed current distribution," Radio Sci., vol. 8, pp. 1169-1176, 1973.

\section{Convergence of Local and Average Values in Three-Dimensional Moment-Method Solutions}

MARK J. HAGMANN, MEMBER, IEEE, AND RONALD L. LEVIN

Abstract - Block models using 8, 64, 216, 512, 1000, 1728, and 2744 cubical cells were used to evaluate the local and average specific absorption rate (SAR) for a dielectric cube irradiated by an EM plane wave. All seven models were used in examples for $0.5-\mathrm{cm}$ and $2.5-\mathrm{cm}$ saline cubes at 400 MHz and a 30-cm cube of biological tissue at $27.12 \mathrm{MHz}$. In each example, the solutions using 8 or 64 cells were similar to that for a sphere rather than a cube. Many cells are needed to approximate the sharp variation of the electric field near corners and edges of a dielectric cube.

The heterogeneity of the electric field in an object having corners and edges causes a frequency-independent error (FIE) in addition to the more

Manuscript received October 9, 1984; revised February 25, 1985.

The authors are with the Biomedical Engineering and Instrumentation Branch, Division of Research Services, National Institutes of Health, 13/3W13, Bethesda, MD 20205. 
generally observed frequency-dependent error (FDE) associated with the electrical size of the object. FIE causes the average SAR to converge less rapidly than local values of SAR at locations distant from the corners and edges. An extrapolant is described that corrects for FDE but not FIE in order to estimate the volume average SAR.

\section{INTRODUCTION}

Numerical solutions obtained using block models have had many applications including the evaluation of biological hazards from exposure to electromagnetic fields [1],[2] and geophysical prospecting [3]. Solutions for block models of man have correctly predicted such phenomena as selective heating of the neck [2] and head resonance [4], as well as the enhancement of energy deposition due to the ground and reflectors [5] or by one or more other bodies [6].

Hyperthermia has shown considerable promise for the adjuvant treatment of cancer, but it is essential that the heat be delivered to the body with a high degree of precision [7]. Since there are practical limits to the number of temperature probes used during hyperthermia, it is our belief that quantitative treatment of deep-seated tumors requires the use of accurate electromagnetic and thermal modeling for estimation of the dose away from the few points that are monitored. We intend to use three-dimensional block models with considerably more cells than have been used previously to provide the high accuracy and increased detail required in this application.

It appears to be desirable to use a pulse-function basis [8] with block models of man since this basis allows the greatest number of cells for a model having maximum detail for realistic representation of the human body. Others have suggested that block model solutions may diverge as the number of cells is increased [9]-[11]. It is for that reason that the present study has been made to test the accuracy and stability of block model solutions. We believe that the examples presented in this paper demonstrate that it is possible to obtain high accuracy with block model solutions if sufficient care is used in their implementation.

\section{Numerical EXAMPLes}

Dielectric cubes were chosen for all examples since the cubical shape allows many different discretizations so that various solutions may be compared to test for convergence. In all examples, the dielectric cube was irradiated by a plane wave with vertical $(Z)$ polarization propagating in the negative $X$-direction where the coordinates are defined in Fig. 1 . The plane wave was defined to have a time-average power density of $1 \mathrm{~mW} / \mathrm{cm}^{2}$. A total of seven solutions were obtained in each example by dividing each edge of the cube into $2,4,6,8,10,12$, and 14 equal sections for a total of $8,64,216,512,1000,1728$, and 2744 cubical cells, respectively. Both local and volume average values of the specific absorption rate (SAR defined as the rate of energy deposition per unit mass) were determined. Considerable computational effort was required in the present study. Even though we used three planes of symmetry with the models to reduce the required size of the matrices, the computations for each of the block models having 1728 and 2744 cells required approximately 7 and $19 \mathrm{~h}$, respectively, of computer time on a VAX-11/750. With 1728 cells, approximately equal amounts of computer time were spent in forming and inverting the large matrices. Formation of the matrices dominated the computer time when smaller numbers of cells were used.

An exact solution for the dielectric cube is not available, but it is well known that the electric field is highly heterogeneous near corners and edges. For this reason, we may expect that many cells

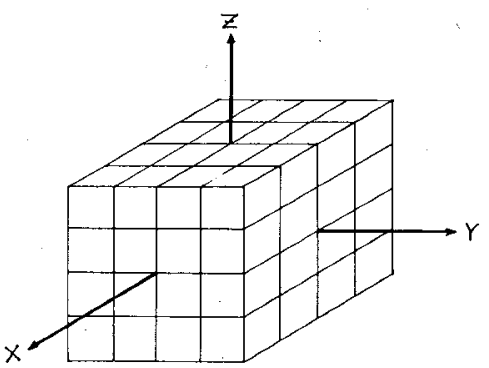

Fig. 1. Coordinates used for irradiation of a dielectric cube by an EM plane wave.

would be needed before accurate values could be obtained near the corners and edges in even the static $(\mathrm{dc})$ case. It is easily shown that the average SAR calculated using one cell as a block model at low frequencies is appropriate for a sphere rather than a cube. Then, since at low frequencies the electric field at the centroid of a dielectric cube is the same as that for a sphere [12], it would be anticipated that the convergence would be much faster at locations near the centroid of the cube than near the corners and edges. The choice of a cubical shape has facilitated the use of multiple discretizations in the following examples, but considerably faster convergence would be expected when modeling smooth objects such as the human body.

Saline was chosen as the dielectric for the first two examples since it has a large dielectric constant, similar to that of most biological tissue, and has been well characterized [13]. The dimensions used in these two examples were chosen so as to permit possible subsequent testing of at least the volume average SAR. It is unfortunate that other solutions for cubes having larger sizes, more convenient for experimental testing with field and temperature probes, appeared to be quite far from convergence. Example 3 was chosen to allow comparison with block model solutions previously described by others [11].

\section{A. Example 1, $0.5 \mathrm{-cm}$ Saline Cube at $400 \mathrm{MHz}$}

The first example was chosen to be a cube of $0.15-N$ saline at $400 \mathrm{MHz}$. $\left(\epsilon^{\prime}=77.1, \sigma=1.40 \mathrm{~S} / \mathrm{m}\right)$ with an edge length $(L)$ of $0.5 \mathrm{~cm}$. For comparison, $k L=0.4181, L / \lambda=0.06269$, and $L / \delta$ $=0.1403$, where $k$ is the magnitude of the complex propagation constant, $\lambda$ is the wavelength, and $\delta$ is the depth of penetration, all three being evaluated in the dielectric.

For each of the seven different discretizations used in each example, there are a number of cubical cells having centroids located along a major diagonal of the dielectric cube. This number is equal to the cube root of $n$, where $n$ is the total number of cells. In Fig. 2, the values of local SAR for all cells located along a major diagonal in each discretization for the first example are given at the locations of their centroids. The extreme right-hand side of the figure corresponds to a location at a front corner of the cube $(X=Y=Z=0.25 \mathrm{~cm})$, and the extreme left-hand side corresponds to a rear corner of the cube $(X=Y=$ $Z=-0.25 \mathrm{~cm})$. In order to minimize confusion, numbers have been used to represent the points rather than using other symbols. Points labeled 1, 2, 3, 4, 5, 6, and 7 correspond to $n=8,64$, $216,512,1000,1728$, and 2744 cells, respectively.

We have used point matching [8] to obtain the matrix elements used in the block model solutions. Since point matching serves to enforce the electric-field integral equation (EFIE) at the cell centroids, we will assume that the calculated values of local SAR are most representative of such points. Others have used electricfield probes to experimentally test the accuracy of calculations made using block models, and their results also suggest that the 


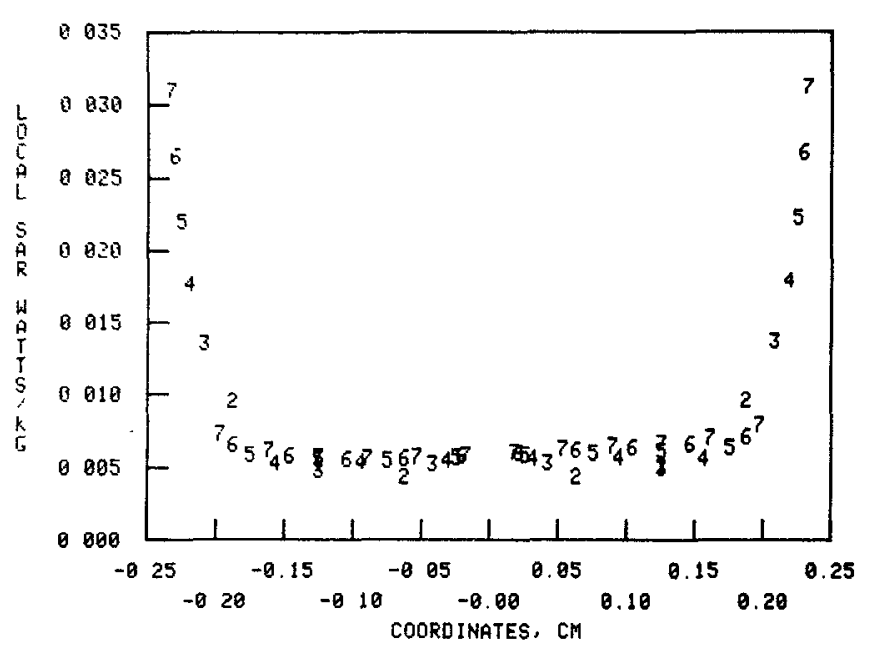

Fig. 2. Local SAR values on diagonal of a $0.5-\mathrm{cm}$ saline cube incident $\mathrm{EM}$ plane wave with $1 \mathrm{~mW} / \mathrm{cm}^{2}$ at $400 \mathrm{MHz} . \epsilon^{\prime}=77.1, \sigma=1.40 \mathrm{~S} / \mathrm{m}$.

values obtained in the solutions are most representative of the cell centroids [14], [15].

Fig. 2 suggests that the values of a local SAR along a main diagonal converge fairly rapidly except at locations near the corners of the cube. For example, the values of local SAR for $n=8,216,1000$, and 2744 at points halfway between the centroid and the corners $(X=Y=Z= \pm 0.125 \mathrm{~cm})$ have ratios of standard deviation to mean of 0.135 and 0.0727 in the front and back, respectively. A small sphere having the same dielectric properties at $400 \mathrm{MHz}$ would have an SAR of $0.004647 \mathrm{~W} / \mathrm{kG}$, which is fairly close to the local SAR near the centroid of the cube as was expected.

The local values of SAR nearest the corners were the largest ones obtained for all seven discretizations. Large values were also obtained near the edges of the cube, especially vertical edges. Since increasing the number of cells causes some points to be sampled that are closer to the corners and edges, greater values of local SAR are obtained at those new locations. By contrast, the centers of the top and bottom faces were found to be the locations with minimum SAR, the local values being somewhat less than half that at the centroid. The reduced value of local SAR near the centers of the top and bottom faces is supported by simple considerations based upon boundary conditions. For all locations in each discretization, the local SAR obtained for a location in the front half of the cube was somewhat greater than that of the corresponding location in the back half, showing the effects of attenuation due to propagation within the lossy dielectric.

The small electrical size used in this example $(k L=0.4181)$ suggests that similar heterogeneous patterns of deposition would be observed for much smaller cubes and even in the limit of zero electrical size. Other calculations made for smaller cubes and for lower frequencies have shown that this is the case. The pattern of deposition in a cube, or presumably in other objects with corners and edges, is highly heterogeneous even in the dc limit. A pulse-function basis is not appropriate if the electric field has appreciable variation within a cell. Two distinctly different phenomena associated with the use of such a basis may be termed frequency-dependent error (FDE) and frequency-independent error (FIE) [16]. It has been known for some time that errors result when such a basis is used unless the cell size is much smaller than a wavelength [17]. This is an illustration of FDE. The present

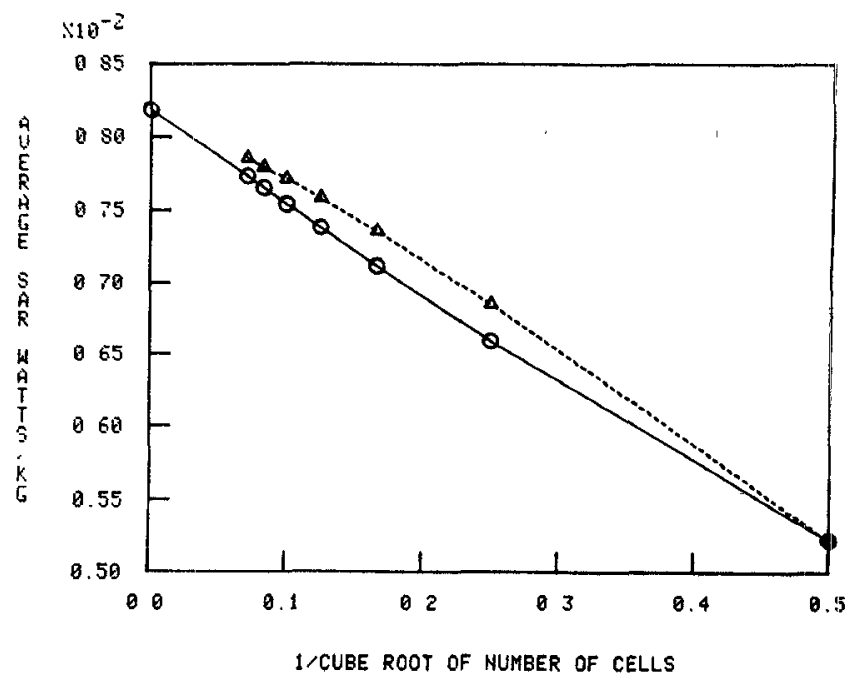

Fig. 3. Average SAR values of a $0.5-\mathrm{cm}$ saline cube incident $E M$ plane wave with $1 \mathrm{~mW} / \mathrm{cm}^{2}$ at $400 \mathrm{MHz} . \epsilon^{\prime}=77.1, \sigma=1.40 \mathrm{~S} / \mathrm{m}$.

data necessitate emphasising the existence of the additional phenomenon of FIE. In some objects, such as the dielectric cube, the electric field has sizable variation even at dc so that there is a minimum number of cells that may be used even for static solutions.

Values of average SAR calculated for each of the seven discretizations of the dielectric cube by simple averaging of the individual SAR values are represented by circles in Fig. 3. A graphical procedure suggested by Mittra and Klein [18] has been used to assist interpreting the extent of convergence. Since a pulse-function basis does not account for even linear variation of the electric field within a cell, it is reasonable that the errors in calculated values of the electric field should be linear in cell size. If these errors are small, then the error in average SAR should also depend linearly upon cell size. Plots with a high degree of linearity, similar to that in Fig. 3, have been obtained using other calculations made using smaller cubes and/or lower frequencies.

It is apparent from Fig. 3 that there is substantial error in the values of volume average SAR in the solutions obtained for the smaller numbers of cells, even though the values of local SAR for those solutions appear to be relatively accurate. Most of the errors in the values of average SAR appear to be due to insufficient sampling of the regions near corners and edges of the dielectric cube where the electric field is highly heterogeneous. Even if the values of local SAR were exact, finite sampling would cause some error in the values of volume average SAR. We have found that an extrapolant is useful in such cases [16]. It is possible to use any two points in Fig. 3 to make an extrapolation to estimate the solution for an infinite number of cells. The line segment extending beyond the data in Fig. 3 is an example of such an extrapolant and was made using the values corresponding to the two largest numbers of cells. There is such a high degree of linearity that the various two-point extrapolations made using the values of average SAR for the four largest numbers of cells $(512,1000,1728$, and 2744$)$ differ from each other by less than 0.01 percent.

Table I gives the calculated values of errors made by using two-point extrapolations when one assumes that the extrapolation made using 1728 and 2744 cells is exact. Table II presents the calculated values of errors for the individual solutions based on the same assumption. While experimental or other confirmation is needed, the results in the two tables suggest that this 
TABLE I

Percentage Errors in Extrapolated Average SAR FOR A 0.5-CM SALINE CUBE

\begin{tabular}{cc}
\hline \hline Numbers of Cells & Percentage Error \\
$8, \quad 64$ & -2.82 \\
64,216 & -0.33 \\
216,512 & -0.07 \\
512,1000 & $<0.01$ \\
1000,1728 & $<0.01$ \\
1728,2744 & $(0.0)$ \\
\hline
\end{tabular}

Final extrapolated value $=0.008184 \mathrm{~W} / \mathrm{kG}$ for plane wave with $1 \mathrm{~mW} / \mathrm{cm}^{2}$.

TABLE II

Percentage Errors in Calculated Average SAR FOR A 0.5-CM SALINE CuBE

\begin{tabular}{ccc}
\hline \hline Total Cells & None & Interpolant \\
8 & -36.1 & -36.1 \\
64 & -19.5 & -16.3 \\
216 & -13.1 & -10.2 \\
512 & -9.8 & -7.3 \\
1000 & -7.9 & -5.8 \\
1728 & -6.5 & -4.8 \\
2744 & -5.6 & -4.1 \\
\hline
\end{tabular}

extrapolation is a highly useful procedure. For example, the solutions obtained using 8 and 64 cells may be combined to allow calculation of the value of volume SAR with greater accuracy than a single solution obtained using 2744 cells that would have considerably greater expense.

Interpolants have also been found to improve the accuracy of values of average SAR by approximating the variation of local SAR at locations other than the sampled points [19]. Since interpolants do not change the local values, they are not useful when the local values themselves have significant error. In the present problem, we have used only the triquadratic interpolant described in [19] and not the NEWSUD, since the NEWSUD will not make the extrapolations required to approximate the higher local SAR values at corners and edges of the dielectric cube. Values of volume-average SAR calculated using the interpolant are represented by triangles in Fig. 3. Note that the interpolant does not just give larger values but the magnitude of the correction decreases when large numbers of cells are used as would be required for convergence to the limit approached without the use of interpolants. It may be seen in Table II that the interpolant makes only a modest correction ( 2 or 3 percent) since it cannot allow for the full sharpness in the variation of the electric-field intensity near the corners and edges of the cube. Interpolants appear to be more helpful when there is less drastic variation, such as in scatterers having smoother shapes.

\section{B. Example 2, 2.5-cm Saline Cube at $400 \mathrm{MHz}$}

The second example was chosen to be a cube of $0.15-N$ saline at $400 \mathrm{MHz}$, with $L=2.5 \mathrm{~cm}$. For comparison, $k L=2.091$, $L / \lambda=0.3135$, and $L / \delta=0.7016$.

Fig. 4 presents the local values of SAR along a major diagonal for all seven discretizations of example 2 using the same notation as described for Fig. 2. The local values for 8 and 64 cells are not significantly different from that of a small dielectric sphere $(0.004647 \mathrm{~W} / \mathrm{kG})$, but for larger numbers of cells there is substantial departure from the solution for a sphere, even near the

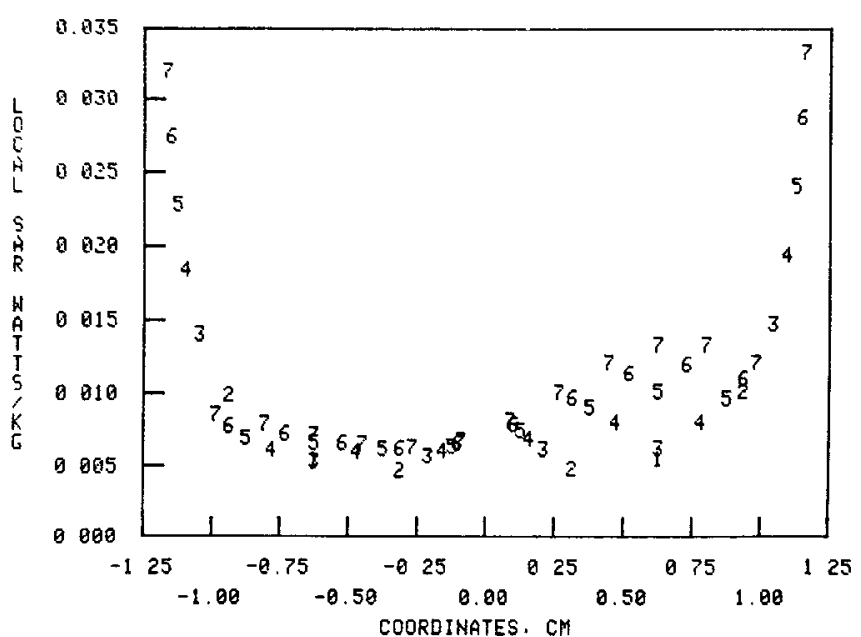

Fig. 4. Local SAR values on diagonal of a $2.5-\mathrm{cm}$ saline cube incident EM plane wave with $1 \mathrm{~mW} / \mathrm{cm}^{2}$ at $400 \mathrm{MHz} . \epsilon^{\prime}=77.1, \sigma=1.40 \mathrm{~S} / \mathrm{m}$.

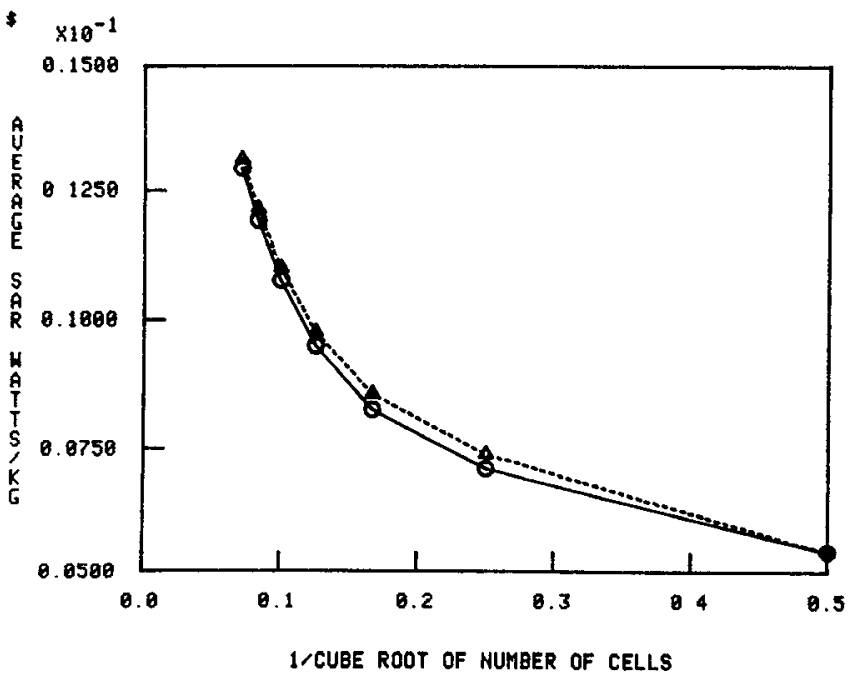

Fig. 5. Average SAR values of a $2.5-\mathrm{cm}$ saline cube incident EM plane wave with $1 \mathrm{~mW} / \mathrm{cm}^{2}$ at $400 \mathrm{MHz}$. $\epsilon^{\prime}=77.1, \sigma=1.40 \mathrm{~S} / \mathrm{m}$

centroid. With the $0.5-\mathrm{cm}$ cube, there was fairly rapid convergence at all locations other than near corners and edges, but for the $2.5-\mathrm{cm}$ cube there is a substantial increase in values of local SAR in the front half of the cube; this increase becomes evident only in the solutions using the largest numbers of cells. By contrast, the values of local SAR near the corners and edges of the dielectric cube appear to be determined mostly by the shape of the object since they are quite close to the values in the first example. As in the first example, the values of local SAR nearest the corners were the largest ones obtained for all seven discretizations.

As in the case of example 1, there is considerable FIE due to insufficient sampling of the rapidly varying fields near corners and edges of the dielectric cube. Since the local values of SAR are similar to those of example 1 in such regions, it is assued that the magnitude of the FIE is comparable to that of example 1 . In example 2, there is a significant amount of FDE as well since some of the local values are appreciably modified by the use of larger numbers of cells. It is important to distinguish between these two sources of error. Limits on the electrical size $(k L)$ of a 


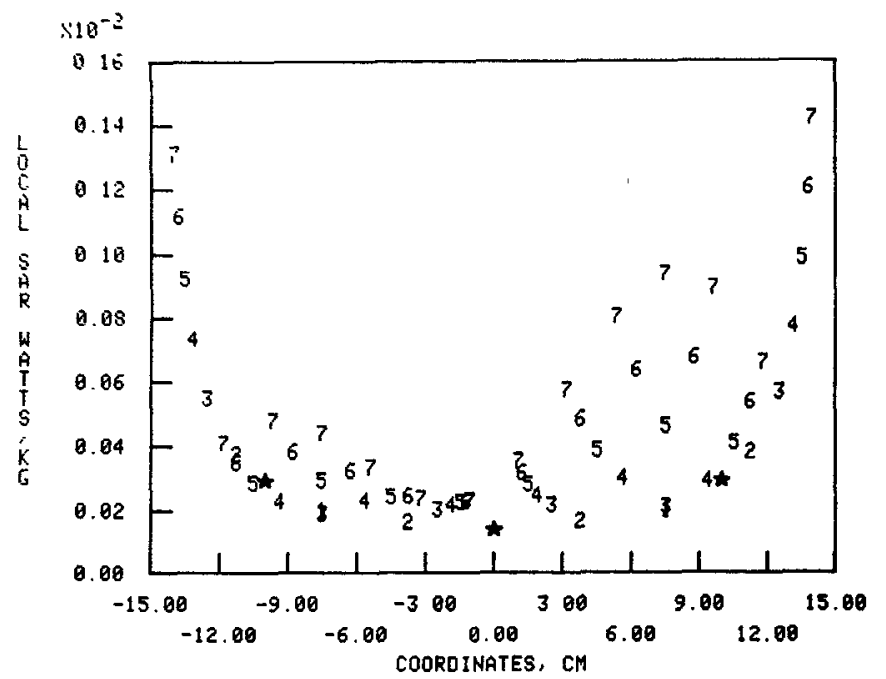

Fig. 6. Local SAR values on diagonal of a $30-\mathrm{cm}$ tissue cube incident EM plane wave with $1 \mathrm{~mW} / \mathrm{cm}^{2}$ at $27.12 \mathrm{MHz}, \epsilon^{\prime}=76.0, \sigma=0.42 \mathrm{~S} / \mathrm{m}$

cell have been presented as a condition that is necessary but not sufficient for convergence [20].

In Fig. 5, values of the volume-average SAR calculated with and without the interpolant are represented by circles and triangles, respectively, as was done in the first example. The variation in Fig. 5 is nonlinear, so it is not possible to estimate the val: of average SAR by extrapolation as was done with the $0.5-\mathrm{cm}$ cube. Attempts to use extrapolants of higher order with these data have also been unsuccessful. We attribute the failure of extrapolation to the presence of FDE. The extrapolant can correct only for FIE, that is, for errors due to insufficient sampling, but requires that the individual samples (values of local SAR) do not have appreciable error. Interpolants are also of little or no use when there is appreciable FDE.

\section{Example 3, 30-cm Cube of Biological Tissue at 27.12 $\mathrm{MHz}$}

The third example was chosen to be a cube of tissue at 27.12 MHz $\left(\epsilon^{\prime}=76.0, \sigma=0.42 \mathrm{~S} / \mathrm{m}\right)$ with $L=30 \mathrm{~cm}$. The parameters of this example were chosen to allow comparison with values previously reported by others [11]. For comparison, $k L=2.897$, $L / \lambda=0.3664$, and $L / \delta=1.758$.

Fig. 6 presents the local values of SAR along a major diagonal for all seven discretizations of example 3 using the same notation as in the two previous examples. In addition, local values given by others [11] for a block model using 27 cells are represented on the same figure by stars. As in the second example, the local values of SAR calculated using small numbers of cells are not significantly different from that of a small dielectric sphere $(0.0001704 \mathrm{~W} / \mathrm{kG})$. In fact, the value obtained for the central cell of a 27 -cell model in [11] differs from this by only 20 percent. The values obtained using larger numbers of cells differ greatly from those for small numbers. In fact, these values suggest that the volume average SAR for the dielectric cube is at least an order of magnitude greater than the value for a small dielectric sphere. The values in Fig. 6 have considerably greater scatter than was observed in the previous two examples. Even away from the corners and edges, the local values of SAR in the front half of the cube appear far from being convergent in the 2744-cell solution. The FDE is much greater than in the other examples as would be predicted from the relatively large electrical size $(k L=2.897)$.

Fig. 7 presents the values of volume-average SAR, as was done in the other two examples. The nonlinear variation in Fig. 7,

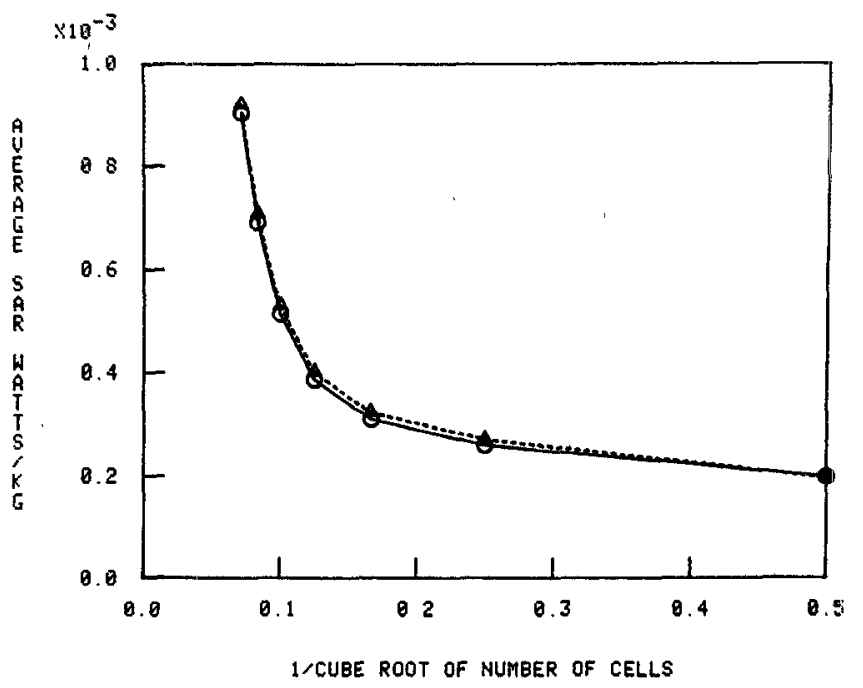

Fig. 7. Average SAR values on a $30-\mathrm{cm}$ tissue cube incident $E M$ plane wave with $1 \mathrm{~mW} / \mathrm{cm}^{2}$ at $27.12 \mathrm{MHz}$. $\epsilon^{\prime}=76.0, \sigma=0.42 \mathrm{~S} / \mathrm{m}$.

which makes extrapolation unuseful, is attributed to inaccuracy of the values of local SAR due to FDE

In [11], the authors presented a 27-cell solution for a dielectric cube using the parameters of this example. They also gave four 34-cell solutions, each obtained by dividing one of the original 27 cells into 8 . Some of the 34-cell solutions differed considerably from that for 27 cells, and the authors concluded that this showed a serious deficiency in the block model formulation [11]. It should be clear from Figs. 6 and 7 that the values in the 27-cell solution are far from being convergent. We are not surprised that they found little change when they subdivided the cell at the centroid of the cube since that location appears to have relatively little correction when 64 cells are used. It is not surprising that subdividing a corner cell caused a substantial increase in the local SAR values. Indeed, all their results with the cube may be attributed to corrections for a solution that is very far frotn convergence.

\section{Conclusions}

It appears that the major source of error in all three examples is the approximation made when assuming that the electric field is a constant within each cell, i.e., the use of a pulse-function basis. We do not believe that significant errors have been introduced by the procedures we used in evaluating the matrix elements since the accuracy of the expressions has been verified by comparison with numerical quadratures. LU decomposition was chosen rather than semi-iterative [9] or other procedures in order to minimize any errors due to matrix inversion for three examples. We do not believe that roundoff errors are significant, despite the large size of some of the matrices used in the present study, since the matrices are diagonally dominant and extremely well conditioned. It is common to use double-precision in the accumulation of scalar products in LU decomposition, as well as forward- and back-substitution in order to limit the buildup of roundoff error. In the present work, we have eliminated the use of double-precision at these steps in several tests using 1728 cells and found that the resulting changes in values of the volumeaverage SAR were less than 3-parts-per-million.

The phenomena of frequency-independent error (FIE) and frequency-dependent error (FDE) have been illustrated by the examples in this paper. In a cube, or presumably in other objects having corners and edges, the electric field has sizable variation 
even in the dc limit so that a large number of cells may be required to determine the average SAR. Since limits which have been specified for the electrical size $(k L)$ of a cell with block models pertain only to FDE [20], such conditions are necessary but not sufficient for convergence.

In calculations made for objects having corners and edges, the volume-average SAR may converge more slowly than values of local SAR at locations distant from the corners and edges. An extrapolant may be used to make substantial corrections for FIE in order to estimate the average SAR with such objects. Extrapolation fails when there is appreciable FDE since the procedure requires that the individual samples of local SAR do not have appreciable error. Interpolants have also been used to make corrections to values of average SAR; but the extrapolant makes a substantially larger correction, so it is the procedure of choice when solutions are available having two or more discretizations. Only values of average SAR obtained without interpolation should be used with the extrapolant since such values have errors which vary linearly with cell size.

We consider it to be essential that the array of cubes used for a block model be arranged for a best-fit of the object to be modeled. Since the electric field has sizable variation near corners and edges, it is necessary that such shapes not be emphasized when modeling a smooth object such as the human body. Increasing the number of cells in a model by subdividing one or more of the cells and retaining the same outer boundary is one way of creating such an emphasis. It appears that when others have used such simple subdivisions with block models of man their results departed from solutions for man and instead approached solutions for models having unwanted corners and edges [9]-[11].

It is anticipated that block model solutions for objects having smooth shapes, such as models of man, will have considerably less FIE than occurs with the dielectric cube so that convergence will be more rapid than is seen in the present examples. In yet-unpublished work, we have found that the values of volumeaverage SAR for block models of a man-sized prolate spheroid at 100 and $225 \mathrm{MHz}$ are within 6 percent of those calculated for the prolate spheroid by other methods. Comparisons of the local values of SAR in both prolate spheroids and spheres with block model solutions for those objects are in progress.

\section{REFERENCES}

[1] M. J. Hagmann, O. P. Gandhi, and C. H. Durney, "Numerical calculation of electromagnetic energy deposition for a realistic model of man," IEEE Trans. Microwave Theory Tech., vol. MTT-27, pp. 804-809, Sept. 1979.

[2] D. E. Livesay and K. M. Chen, "Electromagnetic fields induced inside arbitrarily shaped biological bodies," IEEE Trans. Microwave Theory Tech., vol. MTT-22, pp. 1273-1280, Dec. 1974.

[3] G. W. Hohmann, "Three-dimensional induced polarization and electromagnetic modeling," Geophysics, vol. 40, pp. 309-324, Apr. 1975.

[4] M. J. Hagmann, O. P. Gandhi, J. A. D'Andrea, and I. Chatterjee, "Head resonance: Numerical solutions and experimental results," IEEE Trans. Microwave Theory Tech., vol. MTT-27, pp. 809-813, Sept. 1979.

[5] M. J. Hagmann and O. P. Gandhi, "Numerical calculation of electro" magnetic energy deposition in man with ground and reflector effects," Radio Sct., vol. 14, pp. 23-29, Nov.-Dec. 1979.

[6] O. P. Gandhi, M. J. Hagmann, and J. A. D'Andrea, "Part-body and multibody effects on absorption of radio frequency electromagnetic energy by animals and by models of man," Radio Sci., vol. 14, pp. 15-21, Nov.-Dec. 1979.

[7] G. M. Hahn, "Hyperthermia for the engineer: A short biological prinner," IEEE Trans. Bio-Med. Eng., vol. BME-31, pp. 3-8, Jan. 1984.

[8] R. F. Harrington, Field Computation by Moment Methods. New York: Macmillan, 1968.

[9] J. F. Deford, O. P. Gandhi, and M. J. Hagmann, "Moment-method solutions and SAR calculations for inhomogeneous models of man with large number of cells," IEEE Trans. Microwave Theory Tech., vol. MTT-31, pp. 848-851, Oct. 1983.

[10] E. H. Wissler, "Final report: Thermal and nonthermal effects of microwave radiation," Dept. Chemical Eng., University of Texas at Austın, National Institutes of Health grant GM27844, Mar. 1983.

[11] H. Massoudi, C. H. Durney, and M. F. Iskander, "Limitations of the cubical block model of man in calculating SAR distributions," IEEE Trans. Microwave Theory Tech., vol. MTT-32, pp. 746-752, Aug. 1984.

[12] S. W. Lee, J. Boersma, C. L. Law, and G. A. Deschamps, "Singularity in Green's function and its numerical evaluation," IEEE Trans. Antennas Propagat., vol. AP-28, pp. 311-317, May 1980.

[13] A. Stogryn, "Equations for calculating the dielectric constant of saline water," IEEE Trans. Microwave Theory Tech., vol. MTT-19, pp. 733-736, Aug. 1971.

[14] B. S. Guru and K. M. Chen, "Experimental and theoretical studies on electromagnetic fields induced inside finite biological bodies," IEEE Trans. Microwave Theory Tech., vol. MTT-24, pp. 433-440, July 1976.

[15] K. M. Chen and B. S. Guru, "Internal EM field and absorbed power density in human torsos induced by $1-500-\mathrm{MHz} \mathrm{EM}$ waves," IEEE Trans. Microwave Theory Tech., vol. MTT-25, pp. 746-756, Sept. 1977.

[16] M. J. Hagmann, "Numerical studies of absorption of electromagnetic energy by man," Ph.D. dissertation, Dept. Elec. Eng., Univ. Utah, Salt Lake City, UT, Dec. 1978.

[17] E. M. Purcell and C. R. Pennypacker, "Scattering and absorption of light by nonspherical dielectric grains," Astrophys. J., vol. 186, pp. 705-714, Dec. 1973.

[18] R. Mittra and C. A. Klein, "Stability and convergence of moment-method solutions," in Numerical and Asymptotic Techntques in Electromagnetics, R. Mittra, Ed. New York: Springer-Verlag, 1975, ch. 5.

[19] M. J. Hagmann, O. P. Gandhi, and C. H. Durney, "Improvement of convergence in moment-method solutions by the use of interpolants," IEEE Trans. Microwave Theory Tech., vol. MTT-26, pp. 904-908, Nov. 1978.

[20] M. J. Hagmann, O. P. Gandhi, and C. H. Durney, "Upper bound on cell size for moment-method solutions," IEEE Trans. Microwave Theory Tech., vol. MTT-25, pp. 831-832, Oct. 1977.

\section{$E$-Plane Integrated Parallel-Strip Screen Waveguide Filters}

\author{
FRITZ ARNDT, SENIOR MEMBER, IEEE, JOHANNES BEIKE, \\ DIETRICH GRAUERHOLZ, CHRISTOPH LINGEMANN, \\ AND JENS BORNEMANN
}

Abstract - A rigorous field theory design of a class of rectangular waveguide screen filters is presented which achieves improved attenuation in the upper stopband. The method of field expansion into suitable eigenmodes used considers the effects of the finite rectangular $E$-plane grid thickness and the mutual higher order mode interaction of the single screens. Calculated results up to $55 \mathrm{GHz}$ show that the peak attenuation in the upper stopband for a $\mathrm{Ka}$-band (26-40-GHz) two-resonators filter example with a midband frequency of $f_{0}=37 \mathrm{GHz}$ is about $70 \mathrm{~dB}$, whereas its planar circuit single-metal-insert counterpart reaches only about $34 \mathrm{~dB}$. A $K u$-band (12-18-GHz) filter prototype with three metaletched screens yields a measured passband insertion loss of $0.8 \mathrm{~dB}$ at about $f_{0}=17 \mathrm{GHz}$ and a measured attenuation in upper stopband of about $50 \mathrm{~dB}$ up to $25 \mathrm{GHz}$.

\section{INTRODUCTION}

Metal inserts placed in the $E$-plane of rectangular waveguides achieve low-cost low-loss filter designs [1], [2]. Especially for filters with midband frequencies in the near of the higher band end of the corresponding waveguide housing, the attenuation in the upper stopband attainable with single inserts, however, is often too low [3] for many applications. This is due to unwanted

Manuscript received November 28, 1983; revised February 25, 1985.

The authors are with the Microwave Department, University of Bremen, Kufsteiner Strasse, NW 1, D-2800 Bremen 33, West Germany. 